\title{
OPENINGS IN VERY FORMAL TECHNICAL TEXTS
}

\author{
MICHAEL P. JORDAN \\ QUEEN'S UNIVERSITY AT KINGSTON
}

\section{INTRODUCTION}

The Topic and its Importance

For many technical writers, the openings (title, abstract and introduction) of their work are the most difficult to compose. They are also of great importance because readers need clear statements of the scope, background and objectives of the writing to understand the main discussion and to place conclusions in an appropriate setting. It is therefore useful for us to understand the typical contents, orders and linguistic signalling devices which skilled writers and editors use to introduce their work.

The introductory parts of highly technical (and thus formal) papers are important to writers as well as readers. Before doing the actual technical work (let alone the writing), writers of technical papers need to achieve a firm grasp of the topics to be covered, and to be able to explain and justify their significance and importance. They also need to understand fully: the role of earlier work as it affects the present study, the aim of the work being undertaken, and the methods being used. Writing the title, abstract and introduction helps writers to achieve this understanding. Thus openings in formal technical texts should be regarded not only as essential background information for readers, but also as a valuable way for writers to orient themselves to the major technical and writing tasks facing them. The writing of openings is not the mere communication of already-crystallized thought - it is the means of achieving that necessary goal before spending time and energy on the detailed work and writing. 
Technical students are usually introduced to technical writing by having to write laboratory reports for experiments such as the verification of Ohm's Law, which has been "verified" millions of times before - and to instructors who already know the answers. Although this approach is pedagogically defensible as it provides an introduction to scientific method, it can give students the wrong impression about formal technical papers and reports, which are written to explain real experimental and analytical work. For these, writers have to: introduce (and perhaps define and describe) the topic, explain and justify the importance of the study, describe the methods used for analysis of similar work, relate and critically analyze related work in the literature, identify the specific task that needs to be addressed, and explain how the paper provides some or all of the answers.

These are the roles of introductions in formal technical texts. Introductions are difficult to write because students are rarely required to understand the underlying needs that introductions should fulfil. Even upper-year students are rarely told how to do the important preliminary analysis that is vital to effective original work, and are seldom shown how to introduce their papers and reports. This paper seeks to provide enough background to allow and encourage instructors to fill both these educational voids.

Method Used

This paper is based on the well-known method of corpus-based analysis using published examples of writing. The examples studied for this work have been taken from refereed journals dealing with highly technical topics in a variety of engineering and scientific fields. Some of the work described in the papers studied is stimulated directly by engineering needs, in which a practical solution to a problem needs to be evaluated. Other papers are more scientific in nature, the work involving a search for theory to explain a phenomenon. More frequently, though, the papers represent a combination of engineering and science. For these, the nature of practical engineering problems needs to be understood as the basis for reducing or eliminating the problems. 
Selected extracts from the examples are presented here as examples of the techniques used, and as the basis for the discussion. As the introductions studied are often quite large - typically exceeding several hundred words - it is impractical (and unnecessary) to reproduce them here completely. Thus only selected extracts are included to illustrate the features being described. The selections have been made from commonly-available publications so that readers of this analysis can easily obtain further details if required. Capitalization, indentation, bold type and text placement are reproduced here, but no attempt is made to reflect the type font or size of the original work. Some underlining is added to examples to highlight certain language features for discussion.

Related Work, Specific Task and Contents of this Paper

There is no scarcity of textbook advice regarding the contents and structure of introductions of many types of technical documents. While that advice is usually sound enough, it is a little vague, and all the generalities combined do scant justice to the subject. The comments are also, in total, rather confusing as different writers stress elements they feel are of greater or lesser importance or relevance.

An earlier analysis has dealt in some detail with the titles and introductions of short informal texts (Jordan 1991a), and a second papers analyzes the titles, abstracts and introductions of quite formal texts of considerable (but not fully detailed technical depth (Jordan 1991b). For the informal texts, structures involving problems and their solutions and evaluations dominate the openings, with few exceptions. For the more formal texts, the information structures are more complex, involving not just Problem-Solution structures, but also logical connections of PurposeMeans, Assessment-Basis and Cause-Effect. The first three of these four pairs of relations are also essential elements of openings in very formal technical texts, and in this paper leading capital letters are used to identify logical connections.

Work has also been done to explain abstracts in the formal technical writing of engineering and scientific papers (Jordan 1990). That analysis defines the linguistic genre of technical abstracts, and it also contains 
some preliminary findings on the linguistic features, contents and structures of summaries. The task that remains to be completed here is to analyze the titles and introductions of technical papers, and to place the earlier work on abstracts and summaries within the wider perspective of this present work. As summaries summarize the main body of the paper rather than introduce it, they are not considered part of the specific task here.

The remainder of this paper first explains titles and abstracts for highly technical papers, and then explains the roles and structures of the important elements of introductions: the topic, its importance, the problem, methods used, related work, the specific task, and contents of the paper. This detailed analysis of openings is followed by discussion of "contrasted" pairs of information, a synthesis of the techniques used, and final comments.

\section{ANALYSIS OF OPENINGS}

Titles

Titles for technical papers follow a very simple pattern. Grammatically they are all nominal groups (noun phrases), usually with a deverbal noun (Akimoto, 1989) such as measurement, analysis, or calculation as head. (Deverbal nouns stem from their related verbs measure, analyze and calculate.) Occasionally the head is pre-modified, and is always post-modified, usually in prepositional form. Postmodifications further define the topic or provide detail of the Means by which the work has been done. Here is an example of a simple title:

(1) Analysis of a Radial Groove Gas Face Seal (Tribology Transactions, 35(1), 1992, P11)

The deverbal head noun (Analysis) has no pre-modification, but is postmodified by the prepositional group to provide some definition of the item being analyzed. Within the post-modification, pre-modification (Radial Groove Gas Face) is used to state the specific sub-topic. 
Although titles indicate the topic of the paper, they can rarely give all the detail. For Example 1, further detail is given in the abstract:

(2) An outside-pressurized, bi-directional, gas face seal is analyzed here. This face seal is made up of a hydrodynamic section, consisting of alternate regions of land and groove, and a hydrostatic dam. The grove region...can have either parallel or radial boundaries... (ibid)

Within the post-modifying prepositional group, further postmodifications often occur rather than the pre-modification in Example 1:

(3) Effects of heat treatments on the stress corrosion cracking of alloy 600 in high temperature waters (Nuclear Engineering and Design, 132(2), 1991, P119)

Here prepositional post-modifications are used to further specify the topic of study. The logical connection of Means is commonly included in the title to indicate how the work was done. Here are examples signalled by Through and Using:

(4) Turning Process Identification Through Force Transients (Journal of Engineering for Industry, 114(1), 1992, P1)

(5) Correlation of Thermal Conductivities of Unidirectional Fibrous Composites Using Local Fractal Techniques (Journal of Heat Transfer, 113(5), 1991, P788)

The Means of conducting the work is often included as pre-modification:

(6) Monte Carlo Simulation of Vibrational Relaxation in Nitrogen (Journal of Thermophysics, 6(1), 1992, P22)

(7) p-Version Finite Element Analysis of Gas Bearings of Finite Width (Journal of Tribology, 113, 1991, P417) 
The type of simulation used and the specific method of analysis are included as pre-modifiers. As we shall see later, the Means of conducting the work is often vitally important to the study being described in the paper, and this importance may be reflected in the title.

Many authors are content to use quite a brief title and to provide any necessary elaboration in the abstract and/or introduction. Some, however, try to cram it all in the title:

(8) THE ONSET OF OVERSTABLE MOTIONS IN A LAYER SUBJECTED TO THE SIMULTANEOUS ACTION OF A WEAK UNIPOLAR INJECTION OF CHARGE AND A THERMAL GRADIENT (Journal of Mechanics and Applied Mathematics, 45(1), 1992, P25)

The complexity in the post-modification is made possible by the chain of prepositional phrases and also the restrictive -ed clause dominated by subjected. Although prepositional post-modification is the usual form, restrictive -ed, -ing, and verbless clauses do occur. Here is a simpler example using the -ed clause Immersed...Fluids:

(9) Flexural Vibrations of Rotors Immersed in Dense Fluids (Journal of Fluids and Structures, 6(1), 1992, P3)

Although the noun Vibrations in Example (6) (like treatments in Example 3) is a deverbal noun (based on the verb vibrate), it is different from those in the earlier examples, which derive from cognitive transitive verbs representing what people do in the research (e.g. analyze, identify, and correlate); in contrast vibrate is usually intransitive and inanimate. Thus head nouns in titles are often deverbal nouns related to activities the researchers do during the research. To explain the occurrence of Example 9, we must turn to the abstract:

(10) In this paper, the linear vibrations arising in rotating shafts immersed in a dense annular fluid are analyzed...(ibid)

Thus the writers could have included the deverbal noun Analysis as head noun in their title: 
"Analysis of Flexural Vibrations of Rotors Immersed in Dense Fluids".

Similarly the title

(11) A Computational Procedure for Diffuser-Combustor Flow Interaction Analysis (Journal of Engineering for Gas Turbines and Power, 114, 1992, P1)

is clarified by the words The paper describes in the abstract to indicate that the understood animate deverbal noun is Description.

This approach allows us to understand

ON WOLFE'S LAW OF TRABECULAR ARCHITECTURE (Journal of Biomechanics, 25(1), 1992, P1)

and

(13) CLINICOFUNCTIONAL AND MORPHOLOGICAL CHARACTERISTICS OF THE STATE OF THE STOMACH IN VIBRATION DISEASE PATIENTS (Noise and Vibration Bulletin, 1991, P307)

as being discussions or studies of the law and the characteristics.

In summary, titles of technical papers are nominal groups centred around an implicit or explicit cognitive transitive deverbal noun. They almost always contain detailing post-modification (usually prepositional) and may contain pre-modificational specification. Logical connection of Means may also be included as pre- or postmodification. Details of how titles introduce the topics and sub-topics are given later.

\section{Abstracts and Summaries}

Although the roles and styles of the genres of abstracts and summaries in technical papers have been examined in earlier work, it is useful at 
this point to mention the main findings and to show their relevance to this present study.

Whether labelled "abstract" or "summary" or not labelled at all, the short separate passage of descriptive text immediately following the title is an abstract in the sense that it is used with the title for retrieval purposes. It is also usually an abstract in the sense that it describes what is in the paper (often called "descriptive abstract") rather than describing the work done, or describing or evaluating the topic or the results (often called "informative abstract" or "summary"). In this second sense, an abstract can be recognized as a linguistic genre having two primary language forms:

(a) the paper, work, or author(s) as subject of an active form of a limited number of verbs, such as presents, contains, and outlines; and

(b) the agentless present passive of a larger set of verbs (e.g. is described, are analyzed, is demonstrated), where the implicit agent is the author(s) and the locative (in this paper) is usually also implicit.

Variations of the second (predominant) linguistic form include fronting (e.g. Presented here are), anticipatory It (e.g. It is shown that) and split nominals (e.g. Results are presented which...). It is important to be able to recognize elements of the abstract genre, as this style identifies information about contents of the paper and usually concludes the introduction.

Where the abstract serves only to indicate the contents of the paper (as is usually the case), we can generally disregard it in the analysis of the introduction. This is because the introduction serves other purposes and will be independent of the abstract, repeating or elaborating on any technical detail in the abstract. Similarly, as the title usually indicates only the topic of the paper as a whole, it becomes relevant to our study of the introduction only inasmuch as it provides the key concepts of the presentation. 
However, as some of the papers studied contain information in their abstracts other than the "what is in the paper" group of information (i.e. they are at least partially introductions), it is occasionally necessary to consider their contents in analyzing the introductions - just as we had to turn to the abstract in Examples 1 and 2 to learn of the specific sub-topics, and in Examples 9 and 10 to fully understand the title. In this analysis, information in the abstract is only included in the discussion of introductions when that information is necessary to their understanding.

\section{Introductions - An Introduction}

Introductions (from the Latin ducere - to lead) lead in to or present the main body of the text. To do this they may have to define terms or describe the product being tested or analyzed. The importance and significance of the work often requires explanation, as does the Basis (reasons) for that Assessment of interest and importance. For the technical papers studied here, an explanation of the method(s) used in the work often needs to be included, and details of the related work in the literature are usually necessary. All this leads to the identification of a clearly-defined intellectual (need to know or understand) Problem or task for the work being described; and this in turn leads to details of the contents of the paper (in abstract genre) as a Solution to the intellectual Problem stated.

This is but a brief sketch of the possible contents of an introduction to a technical paper, and the likely order of its presentation. A deeper understanding can be obtained through the analysis of two poor openings which follows. The first is seen to be totally inadequate, while the second contains some of the information necessary for a meaningful opening. For both examples, the titles and abstracts are included here to allow complete analysis. The first example is extremely brief:

\section{Organization of computer-aided design systems}

The possible variants of CAD system structure development is [sic] examined. These variants are based on the main operations of its algorithm functions and differs [sic] in its user characteristics. The 
classification of CAD-systems is carried out based on functional properties.

\section{Introduction}

The operating algorithm of any CAD system can be represented as a set of intercoupled modules. These modules can be realized in hardware and software. The method of implementing the CAD system modules influences the characteristics of the system. Using various implementations and structures of CAD systems, one can use characteristics such as different speeds of operations, storage requirements, etc. [sic] (Mathematics and Computers in Simulation, 33, 1991, P275)

The obvious reader response to this opening is "So what!!", but it is possible to be more specific and constructive in identifying information which could usefully have been included. The opening could have answered the following questions:

"Why is this topic important (Assessment), and what justification (Basis) can you provide for this evaluation?"

"What methods (Means) did you use to achieve the implementation?"

"Has there been any other work on this or related topics, and if so in what respect is it incomplete or inadequate?"

"What is the specific task (Problem) you have set yourself in doing this work?"

"How does this paper provide answers (Solution) to the identified need?"

In each of these categories, we can identify sub-categories of information which might have provided a more meaningful introduction to the paper. These are included in the concluding review of this paper, which includes a list of typical contents for openings of formal technical papers. 
The next example is a little better:

(15) A New Air Classification Principle for Particle Size Range d > $5 \mu \mathrm{m}$

Requirements for processes for air classification for the particle size range $d>5 \mu \mathrm{m}$ are described. A classification principle and first experimental results are presented.

\section{Introduction}

The granulometric state of grinding products is an important quality parameter because of its large influence on the properties of the final products. For example, the influence of the granulometric state can be shown

- for cement...

- for cocoa powders...

- for ceramic powders...

The increasing need for fine powders with definite particle size distributions can be met only partly by direct fine grinding processes because of the stochastic nature of the stress and transport conditions in the mill. Substantially better conditions will be achieved by a combination of grinding and classification. In industry, the classification of the finest powdery materials is mostly realized by air classification. (Particle and Particle Systems Characterization, 8, 1991, P8)

Although the abstract is an incomplete description of the contents of the paper, this introduction does provide some justification for itself by the recognition of an important parameter, and it also provides Basis for that Assessment (signalled by because) and the examples. Although there is no discussion of related work, a Problem is identified by the limitation (met only partly) of one process (Means), followed by a reason for that problem. The final sentence is interesting, but there is no identification of other classification systems or any scholarly justification for using this method. And the connection of air classification in the title, abstract and introduction could have been made clear. 
This introductory analysis allows us to recognize groups of information which are likely to be necessary in any meaningful opening for technical papers:

- the topic (including definition, description, and sub-topics)

- importance of, and interest in (Assessment), the topic or sub-topic together with justification (Basis)

- methods (Means) of doing the practical work or analyzing the topic

- related work, including its description and evaluation of limitations

- identification of the precise task (Problem) the researcher has chosen to undertake

- statement of the methods used and what the paper achieves (usually in abstract style)

While introductions rarely contain all these types of information, many contain most of them. And although the order of the information in the introduction may not always follow that shown above, it is a common arrangement, which many authors follow. For this reason, the topics identified above are dealt with here in the order presented.

\section{The Topic and Sub-Topics}

The topic is established in the initial part of the post-modification of the title. Further specification of the topic being investigated is often also provided in various forms in the major post-modification, or in pre- or post-modification form. An analysis of some of the titles cited so far illustrates the types of specification and the linguistic devices used to communicate them:

Example 1: As the pre-modifier closer to the head (seal), gas-face indicates the type of seal being analyzed; the more remote pre-modifier (radial groove) details a variation of that type, and specific characteristics are provided in the abstract (see Example 2). 
Example 3: The topic of study is alloy 600 , the feature being examined is stress corrosion cracking. The Circumstance under which the work is being done is identified by in high temperature waters.

Example 4: From the abstract, we learn that the topic is the premodifier Turning Process, and that specific features of this topic are identified.

Example 5: The overall topic is Composites, the first sub-topic is Fibrous Composites, and the specific sub-topic is Unidirectional Fibrous Composites. The features studied are Thermal Conductivities.

Example 6: The topic is Nitrogen, and the feature being studied is Vibrational Relaxation.

Example 7: The overall topic is bearings, and the sub-topic is gas bearings; the special types of gas bearing being studied are those of finite width.

Brief descriptions of topics which are not well known to readers often occur in the introduction:

...honeycomb ponds. These ponds float in an air-filled translucent plastic honeycomb structure on top of a body of fresh water. (Solar Energy, 48(2), 1992, P69)

Where the study concentrates on part of a system, a brief description is useful or essential at the start of the introduction:

(17) 1. Introduction

A near-wall turbulent flow can be divided into two regions: the inner region scales on a viscous length, such as $v / u *$ or the Kolmogoroff scale $\left(\mathrm{v}^{3} / \varepsilon\right)^{1 / 4}$; and the outer region scales on a flow dimension, such as channel half width or a boundary-layer thickness. The 
peak turbulent energy, and the peak in production of turbulence both occur within the inner region. If we are concerned with modeling wall-bounded turbulent flow, it is essential to represent this region. (Theoretical and Computational Fluid Dynamics, 3, 1991, P1)

The description can be partly in the form of a formally-announced definition of the topic:

(18) 1. Introduction

A plated-through-hole (PTH) is defined as "A hole in which electrical connection is made between internal and external conductive patterns, or both by plating of metal on the wall of the hole" (IPC-T-50, 1988). (Journal of Electronic Packaging, 114, 1992, P8)

Definition of a concept vital to the purpose of the paper may also be necessary in the introduction:

(18) This paper deals with the problem of determining the effective transverse thermal conductivity of fibrous composites. The effective thermal conductivity is defined as the conductivity of an equivalent homogeneous medium, [sic] which exhibits the same steady-state thermal characteristics as the composite material. (Journal of Heat Transfer, 113(1), 1991, P788)

The Importance of the Topic

Contrary to common belief, engineers and scientists rarely conduct research work without first establishing that it is important enough for them to spend their time on. As they usually need to obtain research funds for equipment and graduate student grants, it is also usually necessary for them to convince others of the importance of their work and this importance is often the initial statement of the introduction: 
(20) 1. Introduction

Process identification is important for automation of machining processes. Among the various process parameters, the ones which characterize the state of tool flank wear are of particular importance. Flank wear directly influences the size and quality of the surface (Jetly, 1984), and the information about its state can be used to plan tool changes to avoid scrap and to manipulate the speed and cutting speed in-process to control tool life (Koren and Ulsoy, 1989). (Journal of Engineering in Industry, 114, 1992, P1)

Both the topic and the sub-topic are Assessed as important, and Basis for the importance of tool blank wear is given in the final sentence cited. Further basis for the statements in the second sentence is provided by the references. In this example, no specific linguistic signal of Assessment or Basis is included as the relationship is clear without it. In contrast, the Assessment-Basis relation is signalled in:

(21) 1. Introduction

The reliability assessment of composite generation and transmission systems has been receiving considerable attention during the past few years [1]. The main reason is that, although these systems cause customer interruptions or loss of service quality very infrequently, they can have a major and widespread effect on society and the environment when problems arise. (IEE Proceedings, 139(2), 1992, P81)

Importance is implied by the considerable attention given to this topic, and the reason for this attention is given in the main clause of the second sentence. The subordinate although clause is Basis against the Assessment (a concession), but that is overpowered by the strength of the main clause supporting the Assessment. The Assessment of importance and its Basis often appear within the same sentence: 


\section{(22) 1. Introduction}

With the increasing use of composites in various thermal environments, the thermal properties of unidirectional fibrous composites have been the focus of many investigations in the last few years. (Journal of Heat Transfer, 113, 1991, P788)

The subordinator With (=As a result of) indicates Basis for the Assessment in the main clause. Even closer cohesion between Assessment and Basis is common as starts for the introduction:

\section{Introduction}

INTEREST in vibration-dissociated coupling in diatomic gases is a result of its influence on dissociation rates at hypersonic speeds. (Journal of Thermophysics, 6(1), P22)

The Assessment of importance implied in the nominal INTEREST...gases is given Basis in the complement its...speeds, with the complex verb structure is a result of (=results from) providing the signalling.

Although researchers are often dealing with very practical problems, importance may derive from an intrinsic intellectual interest in understanding the nature of a problem or how something works. It is thus useful to be able to distinguish between practical and intellectual importance, as noted in:

(24) The ensuing interdisciplinary phenomena are obviously of intrinsic interest for their behavioural richness in extending the applications of instability and associated bifurcation theories, but they have also been studied for more practical purposes. (Journal of Mechanics and Applied Mathematics, 45(1), 1992, P25)

The Problem and the Means of Solving it

An effective way of establishing the importance of the topic is to identify the practical or intellectual problem addressed in the paper: 


\section{INTRODUCTION}

VIBRATIONS ARISING IN ROTATING MACHINERY have long been recognized as an important problem, affecting normal operation and plant reliability... Of particular concern are unstable regimes which may develop under certain conditions, leading to severe rotor vibrations. (Journal of Fluids and Structures, 6, 1992, P3)

In this example, the second sentence cited establishes an important part of the problem the paper seeks to solve or understand. Earlier attempts to solve the identified problem may also be needed to narrow down the problem to be addressed:

\section{INTRODUCTION}

The need for reducing sound radiation from and transmitted through an elastic structure is a common problem. Attempts at achieving moderate levels of noise reduction by passive applications and structural modifications have been inadequate for lower frequencies. The possibility of using active devices for this type of noise control is evolving...(Journal of Sound and Vibration, 153(3), 1992, P387)

We are now involved with the complex set of activities (and related linguistic structures) dealing with problem recognition and identification of the specific intellectual task to be undertaken. This and the next sub-section of this paper discuss some of these complexities. In Example 26, an attempt to solve the problem by a particular Means (by passive applications) is Assessed as being inadequate for part of the problem. Discussion then turns to the use of active devices as the means of solving the problem.

Just as we can recognize practical and intellectual problems, we can also recognize practical and intellectual Means or methods of solving them. In Example 26, engineering methods are used to solve the problem, as also in: 


\section{(27) INTRODUCTION}

Functional electrical simulation (FES) has been demonstrated to be feasible in assisting paraplegic individuals to stand up... (Journal of Biomedical Engineering, 14(1), 1992, P 1)

The intellectual Means (or method or way) by which a need-to-know problem is solved is of at least equal importance for openings. For these, we have to recognize that different methods of analysis or modelling are possible, and that each has its uses, advantages and disadvantages:

(28) In computational fluid dynamics (CFD) three different types of methods are usually employed. The finite volume/difference methods are the simplest and most efficient and...can be applied to complex geometries. Finite element methods can easily be used on irregular geometries, but the equations are more complex and it is often more difficult to explain them physically. Spectral methods have been around for some time, but their applications have mostly been limited to simple problems [1]. In the present work...the finite volume method is selected. (Numerical Heat Transfer, $\overline{21,1992,}$ P1)

It is often necessary to explain deficiencies in one (often the traditional) method of understanding a problem, or to explain why it is not the best method for the particular problem being addressed. The related discussion can often be quite involved, because the author(s) recognizes limitations or difficulties with one method and these are overcome with another method:

(29) Currently these losses are calculated using empirical correlations based on existing experimental data. Such an approach cannot be used effectively for new design concepts. Further, due to the complexity of the geometry and the resulting flow, the choice of the optimum configuration depends upon the selection of a large number of parameters. Evidently, the conventional approach of identifying an optimum combination of a large number of parameters through scale model testing followed by full-scale testing is both expensive and time consuming. These difficulties have led to the 
development of multidimensional computational methods for analyzing the diffuser and combustor external flows. (Journal of Engineering for Gas Turbines and Power, 114, 1992, P1)

Analysis of methods used and the one selected for the present work often dominates the introduction, and many of the references cited indicate work done using one method or another. The following extract is preceded by the opening paragraph of about 250 words dealing almost exclusively with describing the finite element (FE) and finite difference (FD) methods of solving lubrication problems:

(30) In this paper, we formulate gas bearing problems by the p-FE approach previously applied to incompressible bearings (NGuyen, $1990 \mathrm{a} ; 1990 \mathrm{~b}$ ) as opposed to the traditional h-FE formulation used in all the aforementioned FE investigations. The p-FE formulation appears to be a logical choice since the method does not pose much difficulty with high gradient problems (Mehta, 1978). (Journal of Tribology, 113, 1991, P417)

The second sentence of this example provides some Basis for the Assessment to use this particular method for the analysis.

The Specific Task and the Contents

In addition to establishing the topic, its importance, and the method of analysis or problem solving, the opening must also clearly justify and define a specific task. If the task is to establish a better method of analysis, this is achieved through the type of discussion just described. More typically, though, the specific task is justified through an analysis of the literature and a related recognition of a lack of an engineering technique or ability, or a lack of understanding. The lack of an ability is exemplified by:

(31) Consequently, we lack a decisive measurement of the changes in contractility before and after treatment and follow-up investigations of these changes in the same patient over a long period. (Journal of Biomechanical Engineering, 114, 1992, P15) 
This extract follows discussion (Basis) which leads to this Assessment. Where the lack involves understanding, it is usually necessary to explain what work has been done, the limitations and/or deficiencies of that work, and what work has not been done in order to establish the need for the specific task being addressed. References are indicated by [...] in the following extract:

(32) Most of current interest is focused in nonlinear phenomena associated with fluid-film rotor bearings [...], in the response of rotating machinery involving case-strong interaction [...], and in the active control of rotor vibrations [...]. However, to the authors' best knowledge, published work concerning dynamic responses of rotors immersed in dense fields has been rather scarce. Fritz $(1970 a, b)$... was rather incomplete. Indeed no account was given of backward swirl motions, and also no explicit results were presented concerning ... (Journal of Fluids and Structures, 6(1), 1992, P3)

Although such analysis can include disagreements with published literature, it more typically states how the work done fails to provide all the necessary answers to the problem. Indeed authors frequently cite their own work in related topics to establish what has not yet been done:

(33) A previous paper by the authors [2] described the application of sequential simulation to the generation system only, i.e. hierarchical level $I$, and did not include the transmission system. The present paper describes theoretical and computational techniques that include models for the transmission system within the simulation process itself. (IEE Proceedings, 139(2), 1992, P81)

Note that the second sentence here is in "abstract" genre, which is a typical marker of the end of the introduction. Here is a complete paragraph in abstract genre to end an introduction by informing readers of the contents of the paper:

(34) The following deterministic analysis presents the basic mechanism for estimating the unknown surface conditions from the measured interior temperature data. A subsequent statistical analysis 
estimates the effects of measurement error on the estimated results. Finally, criteria for automatically selecting the parameters controlling the sequential procedure was developed. (Journal of Heat Transfer, 114, 1992, P5)

\section{The 'But' Syndrome}

Earlier work on quite formal texts (Jordan, 1991b) identified a group of opening starts dominated by words such as but, however, and although. and we have already seen an example $(\overline{32)}$. The related linguistic structures are also common in the very formal openings studied here, an obvious one being the change from work done to work not done:

\section{INTRODUCTION}

Although there have been numerous studies of the forces transmitted by the human knee during gait, very few of them have attempted to analyze ligament forces (1). This is an important omission in view of the frequency of ligament injury and the increased interest in its surgical treatment. (Journal of Engineering in Medicine, 105, 1991, P11)

The acknowledgement of related work is indicated as "known" by the subordinate clause, and the contrast is signalled by Although to introduce the intellectual need-to-know problem to be addressed. This is Assessed as important, and basis for this Assessment is given in the final clause and signalled by in view of. A similar example in the clearer but form is:

(35) 1. Introduction

The incidence of stress corrosion cracking of Inconnel 600 steam generator tubing has stimulated extensive research aimed at understanding the phenomenon, but a complete understanding of the stress corrosion cracking of alloy 600 in high temperature water has not yet emerged. (Nuclear Engineering and Design, 132(2), 1991, P119) 
Clearly, that is what the paper sets out to achieve. The related structure of concession rather than contrast is also signalled by these words. Concession first provides information of which would lead readers to expect a certain conclusion; but this is followed instead by a statement we would not expect:

(36) According to contemporary ideas, vibration disease is one of the most widespread diseases characterized by polymorphic symptomatics and the involvement in the pathological processes of many organs and systems $/ 2,5,10 /$. However, the functional state of the stomach in people working with handheld vibration prone tools has not been adequately considered in the literature and the results that are available are contradictory /9/. (Noise and Vibration Bulletin, Dec 1991, P307)

From the first sentence we would expect a wealth of research conclusions available on such an important topic. But this is not so, and this lack and the concessive relation are signalled by However.

Transition between traditional ("old") and a possible new method is indicated by However in:

(37) 1. Introduction

Traditional line balancing methods assign one operator to each station on the line. However, the introduction of Japanese manufacturing techniques with the associated philosophy of a flexible work force (Schonberger 1983) have created the opportunity for other methods for manning assembly lines. (International Joumal of Production Research, 30(3), 1992, P469)

Other information pairings typically signalled within these openings are:

(a) one Means is easy for one application, but not for others

(b) an Assessment applies in theory, but not in practice

(c) a transition is indicated between a good and bad Assessment

(d) work is done but is incomplete or inadequate. 


\section{REVIEW}

\section{A Synthesis}

It is now possible to synthesize the typical contents and structures that make up effective openings in very formal technical texts. This synthesis is presented in tabular form in Appendix A as a separate page checklist for instructors, editors and writers. Except if perhaps for the introduction to this present paper (which was deliberately modelled on the contents and order presented here!), we should not expect to find introductions that follow completely the system outlined here.

However, we now have some sound basis by which to judge the effectiveness of introductions. We also now have the means of instructing students both in the important underlying types of information they need to understand before proceeding with their work, and in how they can compose these thoughts into effective openings. We can and should expect writers to include most of the types of information identified here, depending on the relevance of that information for the specific work at hand.

Individual writers will, of course, perceive different features of their work as more important than other features, and will use order and emphasis to reflect these perceptions. Thus, even given the same material, different writers will choose different types of information and in different orders for their openings. Nevertheless, we are now better able to question, with some authority and detail, the effectiveness of apparently weak introductions - and we have the means of helping students to improve their openings.

A Good Example Analyzed

To provide some further synthesis of the discussion in this paper, a final example is included which contains many of the features mentioned: 


\section{Fatigue Crack Growth Laws in Small and Large Cracks and Their Physical Background}

\section{Introduction}

It is well known that most fractures in engineering structures and components are caused by fatigue. The fatigue process is mainly divided into two parts i.e., crack initiation process and its propagation process. The latter process is more significant in service because most fatigue life is spent by the propagation life of cracks, especially small cracks, in both plain and notched specimens. Therefore it is very important to clarify the growth behavior of small cracks from an engineering standpoint of fatigue life prediction and designing an alloy with superior resistance to crack growth. Many studies have been carried out regarding crack growth, and many fatigue crack growth laws have been proposed empirically and theoretically (1). Although all these laws have succeeded to some degree in describing each [sic] results, some equations formally contradict each other and the applicable region is limited.

In the present review, first, the unifying explanation is given against the formal contradiction between the crack growth law for small cracks... [and the law] for long cracks. Then, the significance, the applicability and the limitation of the proposed small crack growth law and the effect of various factors on the law will be discussed.

...The features and the definition of small cracks have been reviewed in many papers (4)-(6). In the present paper we mainly focus our investigation on the small cracks which propagate under high stress... (International Journal, 35(1), 1991, P1)

The topic (small and large fatigue cracks), and the sub-topics (growth laws and physical background) are established in the title. Some known background and description are given in the first two sentences, followed by statements of significance, with the Basis also forming the Basis for the statement of importance for the intellectual challenge. Some detail of the related literature is noted; it is evaluated in a good/bad pair 
dominated by Although, the negative Assessments forming the Problem with the current situation. In abstract genre (is given and the slightly odd future will be discussed), the specific task is noted in a form which overtly informs readers of the contents of the paper. In the final paragraph cited, further details of the literature are mentioned, and the specific task is narrowed down further.

Although as editors we could find cause for some criticism of this opening, it is essentially sound in both contents and order. It tells readers what they need to know at this stage in the paper.

\section{Further Work Needed}

As with many topics of research, this paper poses as many questions as it answers. First, the question of the role of the summary has not been addressed here in a meaningful way. As the summary summarizes the contents of the main body of the paper, it does not provide introductory information, the analysis of which is the main task for this paper. However the role of the summary (especially introductory summaries) does need to be examined in light of the findings presented here.

The similarities and differences between the Problem-Solution pair and the Purpose-Means pair of relations have again been a matter of confusion here as in recent work (Jordan, 1992). Further work is needed to clarify the distinctions or demonstrate that they are really one relation. In addition the horrifying complexities of Problems and Means connections - and the thought processes which stimulate them - need much more attention in language analysis, and perhaps also psychology and pedagogy.

Finally, we need a much greater understanding of closings as well as openings. The two types of conclusion (end and deduction) need to be understood more fully and related to the needs to synthesize and summarize. We also need a greater understanding of the practical and ethical considerations in closings when dealing with the discussion of limitations, defects and the need for clarification or extension. Perhaps much of what is currently included in openings should also be in the closings of earlier work. 


\section{REFERENCES}

Akimoto, M. A Study of Verbo-Nominal Structures in English, The Shinozaki Shorin Press: Tokyo, 1990.

Jordan, M.P. "The Linguistic Genre of Abstracts", The 17th LACUS Forum (A. Della Volpe - ed.), LACUS: Lake Bluff Illinois, 1991 (pp. 507528).

Jordan, M.P. "Openings in Informal Scientific Texts", Technostyle, 9(2), 1991a (pp. 1-19).

Jordan, M.P. "Openings in Quite Formal Scientific Texts", Technostyle, 9(3), 1991b (pp. 18-37).

Jordan, M.P. "An Integrated Three-Pronged Analysis of a Fund-Raising Letter" in Discourse Description: Diverse Linguistic Analyses of a Fund-Raising Text (W.C. Mann and S.A. Thompson - eds.), Benjamins: Amsterdam, The Netherlands, 1992 (pp. 171-226). 


\section{APPENDIX A}

\section{POSSIBLE CONTENTS FOR OPENINGS OF VERY FORMAL TEXTS}

(T-TITLE, A-ABSTRACT, I-INTRODUCTION)

\section{THE TOPIC AND SUB-TOPICS}

Identification of topic

Identification of sub-topic(s)

T/A

Description

Definition

\section{IMPORTANCE OF THE TOPIC}

Statement of importance (Assessment)

Statement of interest or significance (Assessment)

Justification (Basis) for above (including citations)

THE PROBLEM AND MEANS OF SOLVING IT

The technical problem

The intellectual (need-to-know) problem

Earlier solutions (Means) and their evaluation

The Means of solving the technical problem

The Means of solving the intellectual problem

THE SPECIFIC TASK AND THE CONTENTS OF THE PAPER

Earlier work done

Assessment of earlier work together with Basis

Work not done

Statement of specific task

Justification for the specific task

Contents of the paper 


\section{'But' Syndrome Relations}

good/bad assessments (Example 28)

work done/work not done (Examples 32 and 34)

contrast (Example 35)

concession (Example 36)

traditional (old)/new Means (Example 37)

Means for one application/not others

Assessment in theory/not in practice

work done/not complete or inadequate

M.P. Jordan, Learned Conferences, Charlottetown PEI, 1992 